\title{
Strategy in the Search for New Lead Compounds and Drugs from Plants
}

\author{
Kurt Hostettmann*, Andrew Marston, and Jean-Luc Wolfender
}

\begin{abstract}
Plants represent an extraordinary reservoir of novel molecules and there is currently a resurgence of interest in the vegetable kingdom as a provider of new lead compounds for introduction into therapeutic screening programs. Plant constituents of interest are usually isolated following a bioactivity-guided fractionation procedure. In order to render this approach more rapid and efficient, the dereplication of crude plant extracts with LC-hyphenated techniques (LC/UV-DAD, LC/MS and LC/NMR) represents a strategic element to avoid finding known constituents and to target the isolation of new bioactive compounds. The development of simple and rapid bioassays is also crucial for an efficient localisation of active principles. In this respect, the use of bioautographic methods, which enable a direct screening of bioactive constituents on TLC is of great interest. The combination of chemical and biological screening has proved to be very efficient for the survey of an important number of extracts and for the discovery of new lead compounds and several examples of this approach will be discussed in this paper.
\end{abstract}

Keywords: Bioassays $\cdot$ LC/MS $\cdot$ LC/NMR $\cdot$ Lead compounds $\cdot$ Medicinal plants

\section{Introduction}

For centuries plants were virtually the sole therapeutic agents available to humans. With the development of medicinal chemistry in the early nineteenth century, plants were also the first source of substances to be developed as drugs. Nowadays, in spite of the tremendous development of synthetic pharmaceutical chemistry and microbial fermentation, $25 \%$ of prescribed medicines in industrialized countries are of plant origin and some 120 plant-derived compounds from $\mathrm{ca}$. 90 plant species are used in modern therapy. In fact, it is estimated that natural products are implicated in the development

\footnotetext{
${ }^{\star}$ Correspondence: Prof. K. Hostettmann School of Pharmaceutical Sciences Ecole de Pharmacie Genève-Lausanne Laboratoire de pharmacognosie et phytochimie University of Geneva

Quai Ernest-Ansermet 30

$\mathrm{CH}-1211$ Geneva 4

Tel.: +4122379 3401

Fax: +4122379 3399

E-Mail: kurt.hostettmann@pharm.unige.ch
}

of $44 \%$ of all new drugs. Among the $c a$. 350 '000 plant species on the earth, only a small percentage has been phytochemically investigated and the fraction submitted to biological or pharmacological screening is even smaller. Higher plants are thus a source of millions of natural products, with an almost infinite variety of different structural variations [1]. These molecules often have specific functions and many of them have biological activities which can be of use to humans. They may provide lead compounds for the development of new drugs or they may be indispensable tools in biomedical research. While many are substrates for life processes, there are also toxins, hormones, and molecules with other functions. With the introduction of high-throughput screening protocols, there is the capacity to pass large numbers of compounds through a wide variety of bioassays. Since Nature's architecture provides such an unpredictable range of skeletal types and novel substances, it is of immense value to evaluate as many natural products as possible in order to find sources of new drugs [2].

\section{Approaches for the Discovery of New Drugs from Higher Plants}

In order to find new drugs in plants, it is necessary to screen extracts for the presence of novel compounds and to investigate their biological activities. Once novel compounds are suspected, they are generally isolated in order to have material available for further biological and toxicological testing (Fig. 1).

The path which leads from the intact plant to its pure constituents is long. It involves work which might last anything from weeks to years. In order to rationalize and improve the efficiency of the approach, new strategies both in biological screening and chemical screening have to be developed in order to obtain valid chemical and bioactivity information prior to the isolation work [3].

\section{Biological Screening with Simple Bioautographic Methods}

In order to identify the active principle(s) of a given plant, access to bioassays that will reveal the mode of action of a given natural product is mandatory. The choice of the correct therapeutic target can, however, be complex since the key enzymes involved in a disease have to be identified.

In a phytochemical laboratory it is impossible to have access to all targets and the choice of bioassays has to be restricted to relatively simple and sensitive systems that then bring a rather general idea of the type of activity expected. These types of assays can typically be performed on lower organisms, invertebrates and isolated subcellular systems [2].

At the Laboratory of Pharmacognosy and Phytochemistry (LPP), the array of 


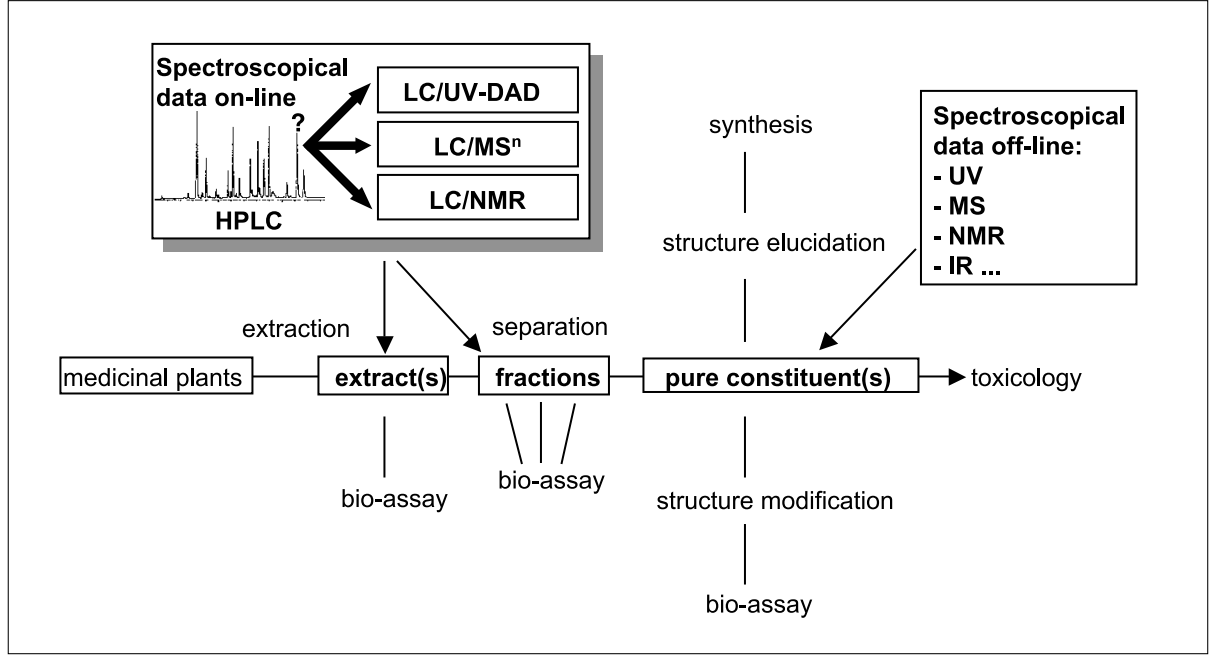

Fig. 1. General scheme for bioactivity-guided isolation. In this process the chemical screening of extracts or fractions with LC hyphenated methods enables the dereplication of known compounds and avoids their re-isolation.

assays includes the testing for antifungal, antibacterial, molluscidical, insecticidal, larvicidal or antioxidant activities [4][5] as well as tests for key enzymes such as acetylcholinesterase (AChE) [6].

In order to obtain a rapid localisation of active natural products directly on TLC, which is important to speed up the isolation process, efforts have been made particularly in the development of bioautography methods [7]. With such methods, the crude extracts, fractions, or pure constituents are resolved on TLC and the organisms or reagents are applied to the TLC plate. Zones of inhibition can be visualized directly on the TLC, thus revealing the activity.

This type of technique has been successfully used for screening antifungal agents inhibiting the growth of a phytopathogenic fungus Cladosporium cucumerinum or the human pathogenic yeast Candida albicans [8].

In our search for new compounds that can be relevant in the pharmacological management of Alzheimer's disease, a bioautographic enzyme assay using AChE has been recently developed [6]. In this test, the enzyme hydrolyses a substrate (naphthyl acetate) and the resulting moiety ( $\alpha$-naphthol) is detected as an azo dye. In the presence of enzyme inhibitors, no hydrolysis occurs and white spots are observed on the TLC plate. The screening of various active plant extracts by this method is shown in Fig 2.

The discovery of bioactivities of extracts and/or fractions by these simple assays is a very useful first step in the process which leads to new therapeutics. This preliminary data must of course be followed up by more thorough in vitro and in vivo testing but the benchtop bioassays are of great value for guiding the whole pathway from the plant to pure new lead compounds.

\section{Chemical Screening with Multihyphenated Methods}

One major drawback of the bioassayguided fractionation strategy is the frequent re-isolation of known metabolites. The chemical screening of crude extracts therefore constitutes an efficient complementary approach allowing localisation and targeted isolation of new types of constituents with potential activities. This procedure also enables recognition of known metabolites at the earliest stage of separation, thus avoiding a time-consuming and costly isolation of common constituents. This process is known as dereplication.

In our laboratory, LC-chemical screening is performed routinely by means of
LC/UV-DAD, LC/MS, and LC/NMR on crude plant extracts (Fig. 1) [9]. The dereplication procedure generally proceeds in two steps. The crude plant extracts exhibiting an interesting bioactivity are first analysed by LC/UV/MS and if complementary structural information is required, de novo structure elucidation can be performed by LC/NMR.

\section{LC/UVIMS Dereplication}

According to the type of products screened, various ionisations can be used (APCI or ESI) and fragment information is obtained by multiple-stage $\mathrm{MS}^{\mathrm{n}}$ experiments performed on-line. For natural products presenting characteristic chromophores, UV spectra are compared to a home-made library of spectra or to literature data. The compounds are also screened by checking if their molecular weights or molecular formulae match with those of known natural products. This type of search usually generates an important number of hits. However, a cross search performed by adding botanical information (genus or family) or the type of constituent reduces drastically the possibilities. The use of a time-of-flight mass spectrometer (TOF) as detector enables the accurate molecular weight assignments of crude extract constituents to be performed on-line and the search can be efficiently restricted in this way [10]. Well-known natural products are thus suitably dereplicated.

\section{LC/NMR Analysis}

If additional information is required, LC/NMR is performed in a second step. This method mainly provides $1 \mathrm{D}{ }^{1} \mathrm{H}-\mathrm{NMR}$ information which is used in combination

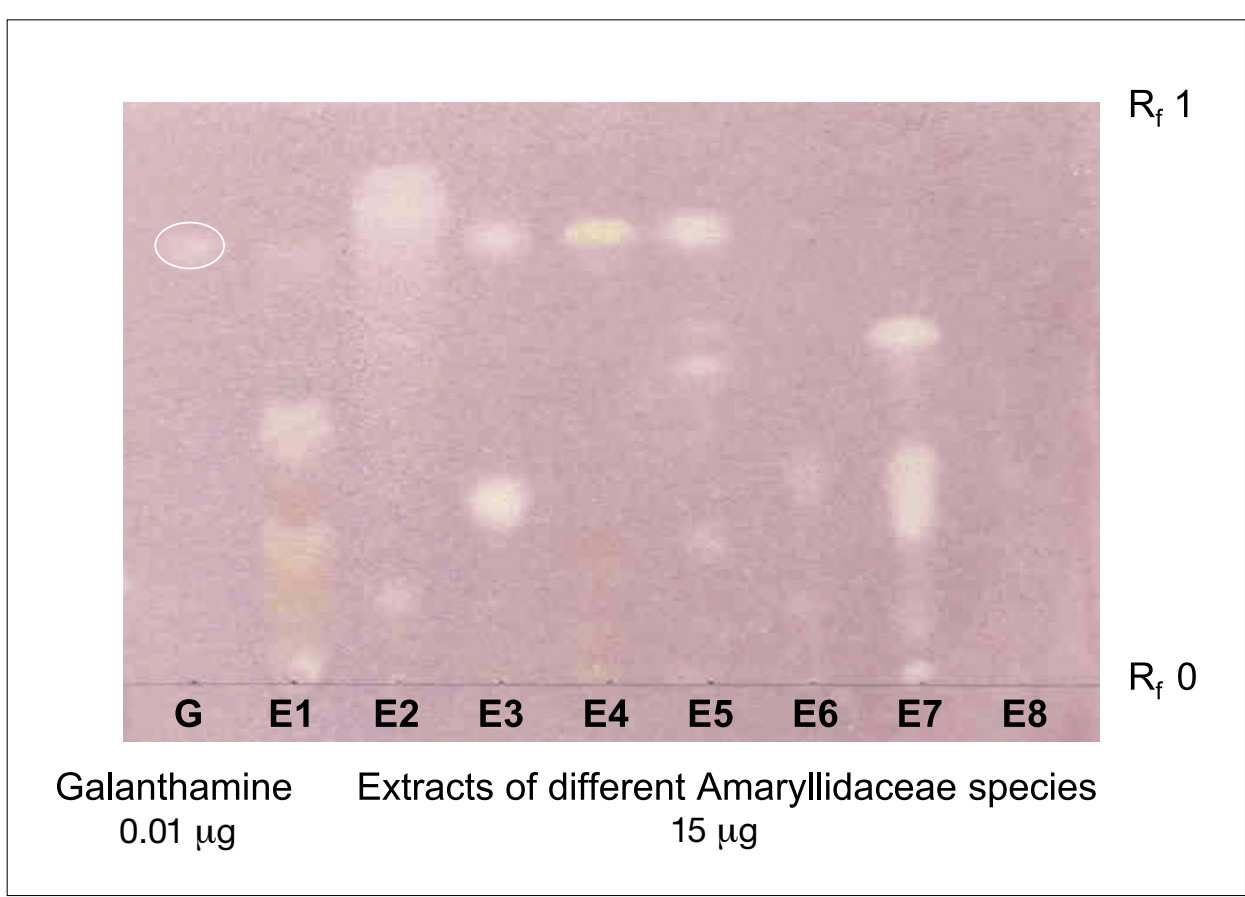

Fig. 2. TLC bioautography for the screening of AChE inhibitors 
with UV and MS or MS/MS for a more specific characterisation of the natural products of interest. LC/NMR is not a sensitive LCdetection technique and the amounts of extracts injected on-column have to be scaled up. Since, for reasons of cost, LC/NMR cannot be operated in fully deuterated solvents, it requires the suppression of signals of standard HPLC grade solvents such as $\mathrm{MeCN}$ or $\mathrm{MeOH}$ [11]. This suppression can be performed automatically by using dedicated pulse sequences [12]. LC/NMR can be operated in two main modes: on-flow or stop-flow. The on-flow mode is generally relatively easy to perform but it is not very sensitive and one way to improve the detection limit is to work in the stop-flow mode. This mode of operation permits longer acquisition times and $\mathrm{LC} /{ }^{1} \mathrm{H}-\mathrm{NMR}$ spectra of compounds present in the low $\mu$ g range can be obtained. In stop-flow, the measurement of various 2D correlations (mainly COSY and TOCSY) is also possible provided that the concentration of the metabolites is high enough. For more sensitive 2D NMR correlation experiments, at-line experiments after microfractionation of the LC-peak of interest in the low microgram range can be performed on a microflow capillary LC/ NMR flow cell with an active volume of $1.5 \mu l$ [13]. In this case NMR spectra are acquired in fully deuterated solvent and no suppression of interfering signals is needed. Thus high quality spectra can be obtained without compromise and demanding experiments such as HMBC can be measured on ca. $40 \mu \mathrm{g}$ of sample (MW 500) overnight.

LC/NMR can be used as a chemical screening technique but it will provide detailed structural information only on the main constituents. It can be used in stopflow mode for the on-line identification of selected compounds and it is also an invaluable technique for studying unstable compounds [14] or compounds not isolable on the preparative scale [15].

Many plants have been screened chemically in our laboratory by LC/UV/MS and LC/NMR and several reviews have summarised the results obtained [9][11][16].

\section{On-line Identification of the Constituents of Erythrina vogelii and Direct At-line Estimation of Their Antifungal Potential}

As an example of the integration of the results of both the biological and chemical screening procedures, the investigation of Erythrina vogelii Hook. f. (Leguminosae), a plant used in the traditional medicine of Ivory Coast to treat various infectious ailments, is presented here [17][18]. This study was motivated by the important activity recorded for the crude dichloromethane root extract in a first screening for antifun- gal properties with the TLC bioautography method, against the plant pathogenic fungus Cladosporium cucumerinum [7].

The extract was firstly analysed by high resolution LC/Q-TOF/MS/MS together with LC/UV-DAD with post-column addition of UV shift reagents. The LC/UV trace revealed the presence of a dozen major peaks. These different constituents shared rather similar types of UV spectra with two main absorption bands of decreasing intensity characteristic for isoflavones. From the high mass accuracy LC/MS data, the molecular formula could be assigned (precision $<5 \mathrm{ppm}$ ) [19]. Presence of fragments due to losses of 56 and $69 \mathrm{Da}$ in the LC/MS/MS spectra of most of the constituents revealed the possible presence of prenyl chains (see Fig. 3E). From these preliminary LC/UV/ MS results, it could be concluded that the $\mathrm{CH}_{2} \mathrm{Cl}_{2}$ extract of $E$. vogelii most probably consisted of a combination of various prenylated isoflavanones or isoflavones.

The on-flow $\mathrm{LC} /{ }^{1} \mathrm{H}-\mathrm{NMR}$ analysis of the extract was carried out by injecting a large amount of the extract on column and the sensitivity of detection was enhanced by performing the separation at low flow rate for the accumulation of an important number of transients across the LC-peaks. On the on-flow contour plot of the crude extract the signals of more than ten LC-peaks were efficiently recorded (Fig. 3A).

During LC/NMR analysis, a LC-microfractionation of the different peaks was performed every $10 \mathrm{~min}$ ( $1 \mathrm{ml}$ fractions) for the antifungal bioautography assays against $C$. cucumerinum. The post-chromatographic antifungal assay revealed that the fractions associated with the LC-peaks eluting at 8.4, $10.8,11.3,12.2$, and $14.6 \mathrm{~h}$ in the low-flow LC/NMR experiment display distinguishable antifungal activities (Fig. 3B).

The ${ }^{1} \mathrm{H}-\mathrm{NMR}$ data extracted from the low-flow LC/NMR analyses confirmed that all the major constituents of the crude extract of E. vogelii were prenylated isoflavones and isoflavanones (Fig. 3A). A first evaluation of the on-flow LC/NMR contour plot revealed that in all chromatographic peaks, aromatic signals between 5.9 and 6.4 ppm were indicative of an A-ring oxygenated in positions 5 and 7 . This was in good agreement with chemotaxonomic data which indicated a 5,7,4' oxygenation for biosynthetic reasons. For the more polar constituents, methylene protons at $4.39-4.57 \mathrm{ppm}$ were characteristic for an isoflavanone nucleus while most of the peaks eluting in the second half of the chromatogram displayed aromatic singlets at $c a .8 \mathrm{ppm}$, which was indicative for isoflavones. The presence of two groups of flavonoids was also confirmed by the presence of two distinct types of chromophores in the UV-DAD spectra recorded. Signals of prenylated groups were detected between 3.0-3.3 ppm (H-1"), 5.1-
5.3 ppm (H-2") and 1.5-1.6 ppm $\left(2 \mathrm{CH}_{3}\right)$ for most of the compounds. As an example, the on-line data obtained for isoflavone 1, which presented the strongest zone of inhibition in the post-chromatographic antifungal bioautography assay, are presented in Fig. 3. Its molecular formula of $\mathrm{C}_{20} \mathrm{H}_{19} \mathrm{O}_{5}$ was deduced from LC/APCI-TOF-MS $(\mathrm{m} / \mathrm{z}$ $339.1208[\mathrm{M}+\mathrm{H}]^{+}$) (Fig. 3D) while losses of $\mathrm{C}_{4} \mathrm{H}_{7}$ or $\mathrm{C}_{5} \mathrm{H}_{9}$ were characteristic for a prenyl moiety in the MS/MS spectrum of the $[\mathrm{M}+\mathrm{H}]^{+}$ion (Fig. 3E). In the on-flow $\mathrm{LC} /{ }^{1} \mathrm{H}-\mathrm{NMR}$ spectrum a singlet at $\delta 7.9$ was assigned to the $\mathrm{H}-2$ proton, characteristic of an isoflavone nucleus. The substitution pattern could be deduced from the aromatic signals $(\delta$ 6.0-7.5) (Fig. 3C). The position of the three hydroxyl groups and the prenyl unit was deduced from the complementary UV-shifted spectra and the MS/MS experiments. The shifted UV spectra (Fig. 3F) were obtained by LC/UV-DAD analyses of the extract with post-column addition of UV shift reagents [20] and analysed according to well-established rules for pure flavonoids [21]. For 1 the 5,7-dihydroxylation of the A-ring was deduced from spectra recorded with $\mathrm{AlCl}_{3}$ and $\mathrm{NaOAc}$ (Fig. 3F). The CID MS/MS spectra obtained, confirmed the presence of two hydroxyl groups on the A-ring (RDA cleavage $\mathrm{m} / \mathrm{z}$ 153.0450 [A1]+) [10] (Fig. 3E). All these data suggested that 1 was isowighteone, a known prenylated isoflavone which was dereplicated by this means [17].

De novo structure determination based on complementary sets of on-line data obtained was performed for each LC-peak of interest and eight polyphenols were thus partially or totally identified on-line. Some of these compounds were found to be new natural products and their targeted isolation was undertaken [17].

\section{Conclusion}

Plants still represent a huge reservoir of interesting bioactive lead compounds for the development of therapeutics. In order to valorise this potential and accelerate the bioactivity-guided isolation process, advanced screening procedures involving both biological and chemical aspects have continually to be developed.

These techniques will also improve the quality and safety control of herbal preparations and precise metabolite profiling of the extracts will become possible. Among the new and exciting challenges which await phytochemists, it is safe to assume that these screening strategies will take an increasingly active part in the discoveries that have yet to be made in natural products chemistry. In parallel to recent progress made in proteomics and genomics, with this technology phytochemists will play an important part 


\section{On-line information obtained for the main antifungal agent}
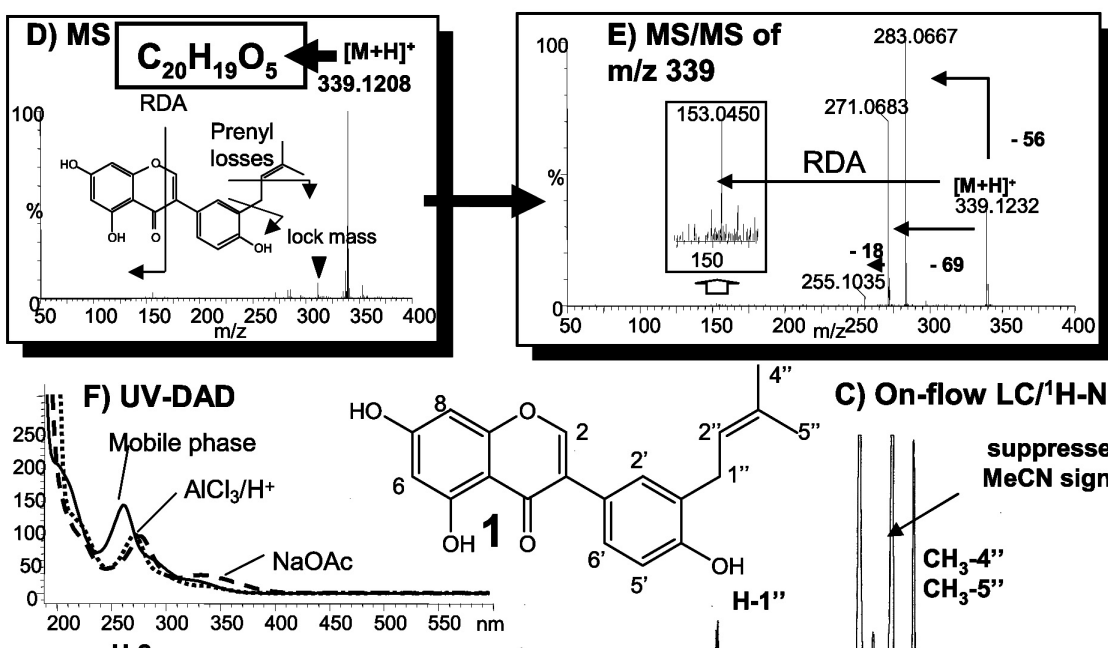

C) On-flow LC/'H-NMR

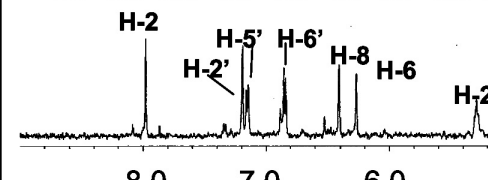

7.0

5.0

Time [hours] A) On-flow LC/NMR and at-line antifungal detection

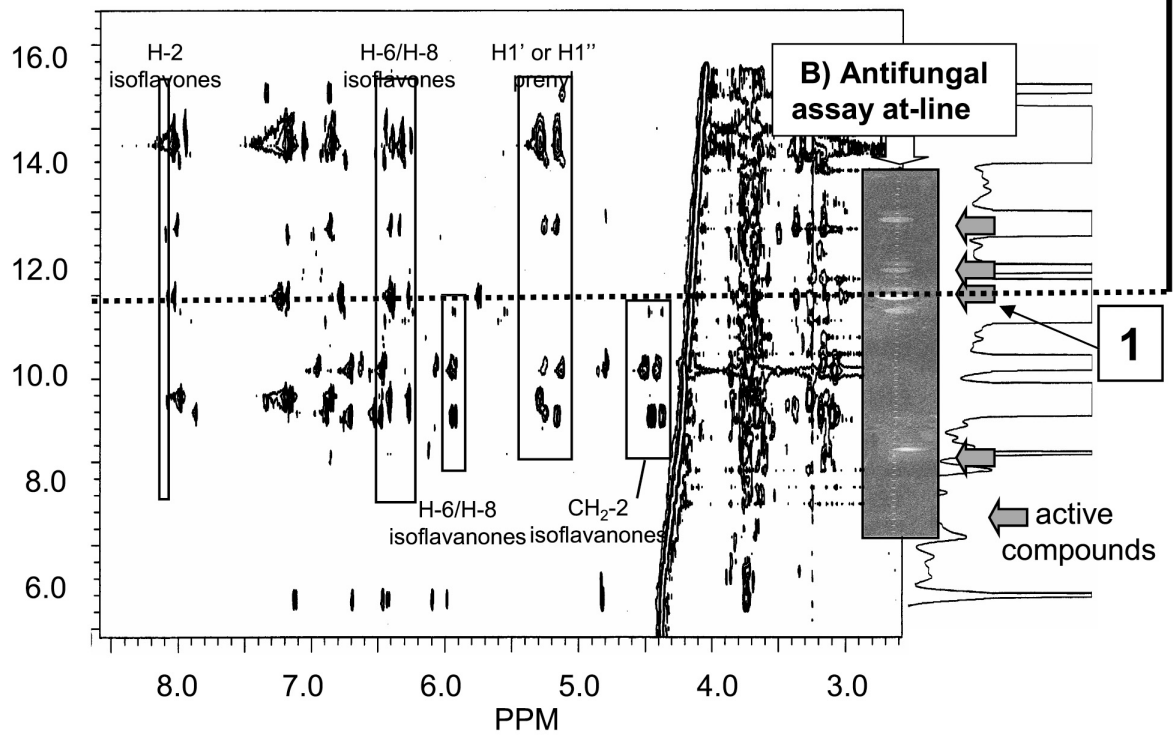

Fig. 3. A) On-flow LC/NMR contour plot of the crude $\mathrm{CH}_{2} \mathrm{Cl}_{2}$ extract of Erythrina vogelii (Leguminosae) showing the ${ }^{1} \mathrm{H}-\mathrm{NMR}$ resonances of all the main constituents. Several characteristic regions for the resonances of isoflavanones and isoflavones are highlighted. B) Inset with the result of the antifungal assay performed on the LC-microfraction against Cladosporium cucumerinum. C) On-flow LC/ ${ }^{1} \mathrm{H}-$ NMR spectrum of the main antifungal agent isowighteone (1) (NT 265). D) LC/Q-TOF/MS spectrum of 1 (calc. mass 333.1232 for $\mathrm{C}_{20} \mathrm{H}_{19} \mathrm{O}_{5}$, error $-2.5 \mathrm{mDa}$ ) E) LC/Q-TOF/MS/MS of $\mathrm{m} / \mathrm{z} 339$ showing typical loss for prenyl moieties and a RDA fragment characteristic for a A-ring with two hydroxyl groups. F) UV DAD spectra of 1 after post-column derivatisation with $\mathrm{AlCl}_{3}$ and $\mathrm{NaOAc}$; shifts are characteristic for a 5,7-dihydroxylation of the A-ring. HPLC conditions: Inj. $10 \mathrm{mg}$; Col. $\mu$-Bondapak C-18 (100 x 8 mm i.d., $10 \mu \mathrm{m}$ ); MeCN-D2O gradient (5:95 to 100:0; 19 hours); 0.05\% TFA; $0.1 \mathrm{ml} / \mathrm{min}$. LC/NMR conditions: 256 scans/increment, flow cell $(60 \mu \mathrm{l}, 3 \mathrm{~mm}$ i.d.), $500 \mathrm{MHz}$. in the new area of large scale metabolite profiling known as metabolomics.

\section{Acknowledgements}

Financial support was provided by the Swiss National Science Foundation (Grant no. 2000100083 to K. Hostettmann)

Received: April 14, 2005

[1] T. Henkel, R.M. Brunne, H. Müller, F Reichel, Angew. Chem. Int. Ed. 1999, 38, 643.

[2] K. Hostettmann, C. Terreaux, Chimia 2000, 54, 652 .

[3] K. Hostettmann, J.-L. Wolfender, C. Terreaux, Pharm. Biol. 2001, 39, 18.

[4] C. Bergeron, A. Marston, R. Gauthier, K. Hostettmann, Int. J. Pharmacogn. 1996 , 34, 233.

[5] M. Cuendet, K. Hostettmann, O. Potterat W. Dyatmiko, Helv. Chim. Acta 1997, 80 , 1144.

[6] A. Marston, J. Kissling, K. Hostettmann, Phytochem. Anal. 2002, 13, 51.

[7] L. Rahalison, M. Hamburger, K. Hostettmann, M. Monod, E. Frenk, Phytochem. Anal. 1991, 2, 199.

[8] L. Rahalison, M. Hamburger, M. Monod, E. Frenk, K. Hostettmann, Planta Med. 1994, 60, 41.

[9] J.-L. Wolfender, K. Ndjoko, K. Hostettmann, J. Chromatogr. A 2003, 1000, 437.

[10] J.-L. Wolfender, P. Waridel, K. Ndjoko, K.R. Hobby, H. Major, K. Hostettmann, Analusis 2000, 28, 895

[11] J.-L. Wolfender, K. Ndjoko, K. Hostettmann, Phytochem. Anal. 2001, 12, 2.

[12] S.H. Smallcombe, S.L. Patt, P.A. Keiffer, J. Magn. Reson. A 1995, 117, 295.

[13] D.L. Olson, J.A. Norcross, M. O’NeilJohnson, P.F. Molitor, D.J. Detlefsen, A.G. Wilson, T.L. Peck, Anal. Chem. 2004, 76, 2966.

[14] A.L. Cogne, E.F. Queiroz, J.-L. Wolfender, A. Marston, S. Mavi, K. Hostettmann, Phytochem. Anal. 2003, 14, 67.

[15] J.-L. Wolfender, L. Verotta, N. Fuzzati, I. Strepponi, K. Hostettmann, Phytochem. Anal. 2003, 14, 290.

[16] J.-L. Wolfender, S. Rodriguez, K. Hostettmann, J. Chromatogr. 1998, 794, 299.

[17] K.K. Atindehou, E.F. Queiroz, D. Terreaux, D. Traore, K. Hostettmann, Planta Med. 2002, 68, 181.

[18] E.F. Queiroz, K.K. Atindehou, D. Terreaux, A. Sandor, K. Hostettmann, J. Nat. Prod. 2002, 65, 403.

[19] E.F. Queiroz, J.-L. Wolfender, K. Atindehou, D. Traore, K. Hostettmann, J. Chromatogr. A 2002, 974, 123.

[20] J.-L. Wolfender, K. Hostettmann, J. Chromatogr. 1993, 647, 191.

[21] K.R. Markham, 'Techniques of Flavonoid Identification', Academic Press, London, 1982 